\title{
Graveyards angiosperm diversity of Rajshahi city, Bangladesh with emphasis on medicinal plants
}

\author{
A. H. M. Mahbubu Rahman \\ Department of Botany, University of Rajshahi, Rajshahi-6205, Bangladesh
}

Email address:

ahmmahbubur_rahman@yahoo.com

\section{To cite this article:}

A. H. M. Mahbubur Rahman. Graveyards Angiosperm Diversity of Rajshahi City, Bangladesh with Emphasis on Medicinal Plants. American Journal of Life Sciences. Vol. 1, No. 3, 2013, pp. 98-104. doi: 10.11648/j.ajls.20130103.14

\begin{abstract}
Angiosperm diversity in the graveyards of Rajshahi city corporation area has been studied. A total of 106 angiosperm species under 91 genera and 44 families were recorded. Of these, Magnoliopsida is represented by 96 species under 82 genera and 40 families while Liliopsida is represented by 10 species under 10 genera and 4 families. Moraceae is the largest family in Magnoliopsida represented by 6 species, and Arecaceae is the largest family in Liliopsida represented by 4 species. Habit analysis shows that herbs, shrubs, climbers and trees are represented by 27, 26, 9 and 44 species, respectively. Forty nine (49) medicinal plants have been documented with their uses for the cure of more than 45 diseases, and some of these are abscess, asthma, abortion, cough, cold, chicken pox, constipation, dysentery, diarrhea, diabetes, eczema, fever, and fracture of bone, headache, heart disease, itches, jaundice, menstrual disease, paralysis, piles, skin diseases, snake-bite, sex problems, toothache, vomiting, worm, wound and others.
\end{abstract}

Keywords: Angiosperm Diversity, Graveyards, Medicinal Plants, Rajshahi, Bangladesh

\section{Introduction}

Rajshahi is one of the oldest towns of Bangladesh. It was promoted to a district headquarter in 1772, a municipality in 1876, a city corporation in 1987 and a metropolitan city in 1992. The city has grown from a small population of 40,000 in 1951 to about 339,932 in 2001. Although Rajshahi was a Divisional headquarter during British period, partition of India in 1947, resulting continuous migration from the Indian side led to sudden population boom in the city. This is the fourth largest city in Bangladesh after Dhaka, Chittagong and Khulna. The city is famous for its high number of educational institutions and situated on the Bank of river Padma. The city is located between $24^{\circ} 21^{\prime}$ north latitudes and between $88^{\circ} 28^{\prime}$ and $88^{\circ} 38^{\prime}$ ' east longitudes.

The city has a sub-tropical monsoon climate, typical of Bangladesh, which falls within a low rainfall zone of the country. 75 percent rainfall occurs during June-September. The annual rainfall is $1350 \mathrm{~mm}$. Temperature of the area is low in January varies from $9.0^{\circ} \mathrm{C}$ to $14.1^{\circ} \mathrm{C}$. From February an increasing trend of temperature is found up to April and thereafter temperature start to decline. In April temperature varies from $22.6^{\circ} \mathrm{C}$ to $36.9^{\circ} \mathrm{C}$. The mean relative humidity is found to be low in March (65\%) and high in JulySeptember (88-89\%) [2].

In every religion they have some sacred places for whom they take care and try to keep them out from external disturbances. The Muslim graveyards are one of such places. The graveyards are situated in such a place, which is outside human habitation and usually have some protected devices. The soil of graveyards is fertile due to the decomposition of human bodies. The places are undisturbed as a result plant can grow at their will. In some cases particular types of plants are planted The management and types of plants grow depend upon the socio-economic pattern of the area[10].

The plants of Rajshahi city corporation area exhibit diverse habitats, such as wetland, scrub jungles, fallow lands etc. which support luxuriant formation of angiosperms and play a vital role in the local economy, environment and primary healthcare system. The importance of studying local floristic diversity has been realized and carried out in Bangladesh by Khan et al.[5], Rahman and Hassan[11], Uddin and Rahman[16], Khan and Huq[6], Uddin and Hassan[17]. Recently Tutul et al.[14, 15] and Rahman et al.[12, 13] conducted the inventorying of angiosperm diversity of Dhamrai Upazilla of Dhaka and Runctia Sal forest of Bangladesh. The 
present study will be made an inventory of the graveyard angiosperm species diversity of Rajshahi City Corporation and to document the medicinal uses of plant species by the local people.

\section{Materials and Methods}

All the specimens mentioned have been collected from three graveyards (Tikapara, Hatemkhan and Meherchandi) of Rajshahi city. A total of 106 species under 92 genera belonging to 44 families were collected and identified. Identification has been confirmed by consulting standard literatures [4], [7], [9] and matching with identified herbarium specimens at RUH and Bangladesh National Herbarium (DACB). Nomenclature has been updated following recent literatures including Ahmed et al. [1] and Pasha and Uddin [8]. The families are arranged (Table 1) according to Cronquist [3], and the species listed alphabetically under each family along with their local name, habit, phenology and voucher number. Emphasis has also been given to the economically important species particularly the medicinal plants used as a primary healthcare. The information on the uses of medicinal plants has been gathered through interview of the local people. The voucher specimens are preserved at RUH for future reference.

\section{Results and Discussion}

Angiosperm diversity in the graveyards of Rajshahi city corporation area has been studied. A total of 106 angiosperm species under 91 genera and 44 families were recorded. Of these, Magnoliopsida is represented by 96 species under 82 genera and 40 families while Liliopsida is represented by 10 species under 10 genera and 4 families. Moraceae is the largest family in Magnoliopsida represented by 6 species, and Arecaceae is the largest family in Liliopsida represented by 4 species. Habit analysis shows that herbs, shrubs, climbers and trees are represented by 27, 26, 9 and 44 species, respectively (Table 1).

The number of species was highest in the Tikapara graveyard. The next number of species was present in the Hatem Khan graveyard. The lowest number of species was observed in the Meherchandi graveyard. Analysis of the species diversity showed $92.45 \%$ species were present in Tikapara, $74.52 \%$ were Hatem Khan and $40.56 \%$ plant species present were Meherchandi graveyard (Table 2).

Out of 44 families recorded from the study area, Seven dominant families are Moraceae, Caesalpiniaceae, Mimosaceae, Euphorbiaceae, Rutaceae, Solanaceae, Asteraceae, and Arecaceae. (Table 3) Analysis of the data based on families showed that leading plant species $13.63 \%$ belonged to Moraceae, $11.36 \%$ Caesalpiniaceae, $11.36 \%$ Mimosaceae, 13.63\% Euphorbiaceae, 11.36\% Rutaceae, $13.63 \%$ Solanaceae, $11.36 \%$ Asteraceae and $9.09 \%$ Arecaceae (Table 5).

Table 1. Graveyards angiosperm species of Rajshahi City

\begin{tabular}{|c|c|c|c|c|}
\hline Taxa & Local name & Habit & Flowering and fruiting time & Voucher Specimens \\
\hline \multicolumn{5}{|l|}{ MAGNOLIOPSIDA } \\
\hline \multicolumn{5}{|l|}{ I . ANNONACEAE } \\
\hline 1. Annona squamosa $\mathrm{L}$. & Ata & Tree & F1.\& Fr.: Mar.- May & MR 71 \\
\hline $\begin{array}{l}\text { 2. Polyalthia longifolia Benth. \& Hook. } \\
\text { II . MENISPERMACEAE }\end{array}$ & Debdaru & Tree & F1. \& Fr.: Mar.- Apr. & MR 05 \\
\hline $\begin{array}{l}\text { 3. Stephania japonica (Thunb.) Miers. } \\
\text { III. MORACEAE }\end{array}$ & Akarnandi & Climber & F1. \& Fr.: Jan.-Dec. & MR 100 \\
\hline 4. Artocarpus heterophyllus Lamk. & Kathal & Tree & F1.\& Fr.: Nov.- Mar. & MR 34 \\
\hline 5. Ficus benghalensis L. & Bot & Tree & F1.\& Fr.: Jan.-Dec. & MR 56 \\
\hline 6. Ficus hispida L. f. & Kakdumur & Tree & F1.\& Fr.: Jan.-Dec. & MR 77 \\
\hline 7. Ficus racemosa $\mathrm{L}$. & Dumur & Tree & F1.\& Fr.: Jan.-Dec. & MR 43 \\
\hline 8. Ficus religiosa L. & Pakur & Tree & F1.\& Fr.: Jan.-Dec. & MR 29 \\
\hline $\begin{array}{l}\text { 9. Morus indica } \mathrm{L} \text {. } \\
\text { IV. NYCTAGINACEAE }\end{array}$ & Tut & Tree & F1.\& Fr.: Jan.-Oct. & MR 16 \\
\hline $\begin{array}{l}\text { 10. Boerhaavia diffusa } \mathrm{L} \text {. } \\
\text { V. AMARANTHACEAE }\end{array}$ & Punarnava & Climber & F1.\& Fr.: Feb.-Nov. & MR 57 \\
\hline 11. Achyranthes aspera L. & Apang & Herb & F1.\& Fr.: Jan.-Dec. & MR 25 \\
\hline 12. Amaranthus lividus L. & Noteyshak & Herb & F1.\& Fr.: Jan.-Dec. & MR 22 \\
\hline $\begin{array}{l}\text { 13. Amaranthus spinosus L. } \\
\text { VI. POLYGONACEAE }\end{array}$ & Kantanotey & Herb & F1.\& Fr.: Jan.-Dec. & MR 23 \\
\hline 14. Polygonum barbatum L. & Panimorich & Herb & F1.\& Fr.: Jan.-Dec. & MR 44 \\
\hline 15. Polygonum hydropiper L. & Chotopanimorich & Herb & F1.\& Fr.: Jul.-Sep. & MR 47 \\
\hline 16. Polygonum orientale L. & Boropanimorich & Shrub & F1.\& Fr.: Jan.-Mar. & MR 39 \\
\hline $\begin{array}{l}\text { 17. Polygonum plebejum L. } \\
\text { VII. BOMBACACEAE }\end{array}$ & Raniful & Herb & F1.\& Fr.: Mar.-Aug. & MR 40 \\
\hline 18. Bombax ceiba L. & Simul & Tree & F1.\& Fr.: Jan.-Mar. & MR 50 \\
\hline
\end{tabular}




\begin{tabular}{|c|c|c|c|c|}
\hline \multicolumn{5}{|l|}{ VIII. MALVACEAE } \\
\hline 19. Hibiscus rosa-sinensis $\mathrm{L}$. & Joba & Shrub & F1.\& Fr.: Jan. -Dec. & MR 14 \\
\hline $\begin{array}{l}\text { 20. Thespesia populnea (L.) Sol. ex Corr. } \\
\text { IX. CUCURBITACEAE }\end{array}$ & Parashpipul & Tree & F1.\& Fr.: Jan.- Jun. & MR 70 \\
\hline $\begin{array}{l}\text { 21. Coccinea cordifolia (L.) Cogn. } \\
\text { X. SAPOTACEAE }\end{array}$ & Telakucha & Climber & F1.\& Fr.: May-Oct. & MR 98 \\
\hline $\begin{array}{l}\text { 22. Manilkara zapota (L.) P. Van. Royen } \\
\text { XI. EBENACEAE }\end{array}$ & Sopheda & Tree & F1.\& Fr.: May-Jun. & MR 94 \\
\hline $\begin{array}{l}\text { 23. Diospyros perigrina (Gar.) Gurke. } \\
\text { XII. CRASSULACEAE }\end{array}$ & Deshigab & Tree & F1.\& Fr.: Apr.-May & MR 55 \\
\hline $\begin{array}{l}\text { 24. Kalanchoe pinnata (Lamk.) Pers. } \\
\text { XIII. ROSACEAE }\end{array}$ & Patharkuchi & Herb & F1.\& Fr.: Jun.-Sep. & MR 49 \\
\hline $\begin{array}{l}\text { 25. Rosa centifolia } \mathrm{L} . \\
\text { XIV. FABACEAE }\end{array}$ & Golap & Shrub & F1.\& Fr.: Jan.-Dec. & MR 65 \\
\hline 26. Cajanus cajan (L.) Huth. & Arhar & Shrub & F1.\& Fr.: Oct.-Jan. & MR 23 \\
\hline 27. Dalbergia sissoo Roxb. ex A.P.DC. & Sissoo & Tree & F1.\& Fr.: Feb.-Mar. & MR 61 \\
\hline $\begin{array}{l}\text { 28. Erythrina variegata (L.) Merr. } \\
\text { XV. CAESALPINIACEAE }\end{array}$ & Madar & Tree & F1.\& Fr.: Feb.-Apr. & MR 20 \\
\hline 29. Bauhinia acuminata $\mathrm{L}$. & Kanchan & Tree & F1.\& Fr.: Mar.-Nov. & MR 19 \\
\hline 30. Cassia sophera L. & Kalkasundha & Shrub & F1.\& Fr.: Dec.-Feb. & MR 93 \\
\hline 31. Cassia alata $\mathrm{L}$. & Dadmardan & Shrub & F1.\& Fr.: Jul.-Aug. & MR 52 \\
\hline 32. Delonix regia (Bojer) Raf. & Krishnachura & Tree & F1.\& Fr.: Apr.-Jun. & MR 60 \\
\hline $\begin{array}{l}\text { 33. Tamarindus indica } \mathrm{L} \text {. } \\
\text { XVI. MIMOSACEAE }\end{array}$ & Tetul & Tree & F1.\& Fr.: May-Jun. & MR 22 \\
\hline 34. Acacia nilotica (L.) Willd. & Babla & Tree & F1.\& Fr.: Jun.-Sep. & MR 41 \\
\hline 35. Acacia catechu Willd. & Khair & Tree & F1.\& Fr.: May-Jun. & MR 24 \\
\hline 36. Acacia auriculiformis A. Cunn ex Benth. & Akashmoni & Tree & F1.\& Fr.: Jul.-Sep. & MR 92 \\
\hline 37. Albizia procera (L.) Benth. & Koroi & Tree & F1.\& Fr.: May-Sep. & MR 26 \\
\hline $\begin{array}{l}\text { 38. Mimosa pudica } \mathrm{L} \text {. } \\
\text { XVII. LYTHRACEAE }\end{array}$ & Lajjaboti & Climber & F1.\& Fr.: Aug.-Nov. & MR 69 \\
\hline 39. Lagerstroemia speciosa (L.) Pers. & Jarul & Tree & F1.\& Fr.: Apr.-Jun. & MR 62 \\
\hline $\begin{array}{l}\text { 40. Lawsonia inermis L. } \\
\text { XVIII. MYRTACEAE }\end{array}$ & Mehedi & Shrub & F1.\& Fr.: May-Jun. & MR 74 \\
\hline 41. Eucalyptus camaldulensis Hook. & Eucalyptus & Tree & F1.\& Fr.: Aug.-Oct. & MR 28 \\
\hline 42. Psidium guajava L. & Piyara & Tree & F1.\& Fr.: Jan.-Dec. & MR 36 \\
\hline $\begin{array}{l}\text { 43. Syzygium cumini (L.) Skeel } \\
\text { XIX. PUNICACEAE }\end{array}$ & Kalojam & Tree & F1.\& Fr.: Mar.-May & MR 73 \\
\hline $\begin{array}{l}\text { 44. Punica granatum } \mathrm{L} \text {. } \\
\text { XX. COMBRETACEAE }\end{array}$ & Dalim & Shrub & F1.\& Fr.: Feb.-Mar. & MR 97 \\
\hline 45. Terminalia arjuna (Roxb.) Wt. \& Arn. & Arjun & Tree & F1.\& Fr.: Apr.-May & MR 32 \\
\hline $\begin{array}{l}\text { 46. Terminalia chebula (Gaertn) Retz. } \\
\text { XXI. EUPHORBIACEAE }\end{array}$ & Haritaki & Tree & F1.\& Fr.: Apr.-Aug. & MR 24 \\
\hline 47. Acalypha indica Burm. f. & Muktajhuri & Herb & F1.\& Fr.: Sep.-Jun. & MR 33 \\
\hline 48. Croton bonplandianum Baill. & Bonjhal & Herb & F1.\& Fr.: Jan.-Dec. & MR 66 \\
\hline 49. Euphorbia hirta L. & Dudhia & Herb & F1.\& Fr.: Oct.-May & MR 35 \\
\hline 50. Phyllanthus reticulatus Poir. & Panisitki & Shrub & F1.\& Fr.: May-Jun. & MR 20 \\
\hline 51. Phyllanthus emblica $\mathrm{L}$. & Amloki & Tree & F1.\& Fr.: Mar.-May & MR 27 \\
\hline $\begin{array}{l}\text { 52. Ricinus communis } \mathrm{L} \text {. } \\
\text { XXII. RHAMNACEAE }\end{array}$ & Rendri & Shrub & F1.\& Fr.: Jan.-Dec. & MR 75 \\
\hline $\begin{array}{l}\text { 53. Zizyphus mauritiana Lamk. } \\
\text { XXIII. VITACEAE }\end{array}$ & Boroi & Tree & F1.\& Fr.: Sep.-Nov. & MR 38 \\
\hline 54. Cayratia trifolia (L.) Domin & Banangur & Climber & F1.\& Fr.: Jun.-Sep. & MR 39 \\
\hline $\begin{array}{l}\text { 55. Vitis quadrangularis Wall. } \\
\text { XXIV. SAPINDACEAE }\end{array}$ & Harzora & Climber & F1.\& Fr.: Aug.-Sep. & MR 87 \\
\hline $\begin{array}{l}\text { 56. Litchi chinensis Sonn. } \\
\text { XXV. ANACARDIACEAE }\end{array}$ & Lichu & Tree & F1.\& Fr.: Jan.-Feb. & MR 78 \\
\hline $\begin{array}{l}\text { 57. Mangifera indica } \mathrm{L} \text {. } \\
\text { XXVI. RUTACEAE }\end{array}$ & $\mathrm{Am}$ & Tree & F1.\& Fr.: Jan.-Mar. & MR 31 \\
\hline 58. Aegle marmelos (L.) Correa & Bel & Tree & F1.\& Fr.: Apr.-May & MR 42 \\
\hline 59. Citrus grandis (L.) Osbek. & Jambura & Tree & F1.\& Fr.: Feb.-Mar. & MR 95 \\
\hline 60. Feronia limonia (L.) Swingle & Kothbel & Tree & F1.\& Fr.: Feb.-Mar. & MR 12 \\
\hline 61. Glycosmis pentaphylla (Roxb.) DC. & Atissorah & Shrub & F1.\& Fr.: Nov.-Dec. & MR 59 \\
\hline $\begin{array}{l}\text { 62. Murraya paniculata (L.) Jacq. } \\
\text { XXVII. MELIACEAE }\end{array}$ & Kamini & Shrub & F1.\& Fr.: May-Aug. & MR 45 \\
\hline 63. Azadirachta indica A.Juss. & Neem & Tree & F1.\& Fr.: Mar.-Apr. & MR 38 \\
\hline 64. Melia sempervirens (L.) All. & Beraneem & Tree & F1.\& Fr.: Mar.-May & MR 47 \\
\hline 65. Swetenia mahagoni L. & Mehagoni & Tree & F1.\& Fr.: Mar.-Apr. & MR 58 \\
\hline
\end{tabular}




\begin{tabular}{|c|c|c|c|c|}
\hline \multicolumn{5}{|l|}{ XXVIII. OXALIDACEAE } \\
\hline 66. Biophytum sensitivum (L.) APDC & Panilazuk & Herb & F1.\& Fr.: Sep.-Mar. & MR 68 \\
\hline $\begin{array}{l}\text { 67. Oxalis corniculata L. } \\
\text { XXIX. APIACEAE }\end{array}$ & Amrul & Herb & F1.\& Fr.: Sep.-Mar. & MR 85 \\
\hline $\begin{array}{l}\text { 68. Centella asiatica }(\text { L.) Urban } \\
\text { XXX. APOCYNACEAE }\end{array}$ & Thankuni & Herb & F1.\& Fr.: Feb.-May & MR 49 \\
\hline 69. Alstonia scholaris (L.) R.Br. & Chatim & Tree & F1.\& Fr.: Oct.-Feb. & MR 50 \\
\hline 70. Catharanthus roseus (L.) G.Don & Nayantara & Herb & F1.\& Fr.: Jan.-Dec. & MR 51 \\
\hline $\begin{array}{l}\text { 71. Thevetia peruviana K.(Pers.) Schum. } \\
\text { XXXI. ASCLEPIADACEAE }\end{array}$ & Kolkiphul & Shrub & F1.\& Fr.: Jan.-Dec. & MR 90 \\
\hline $\begin{array}{l}\text { 72. Calotropis procera } \mathrm{R} . \mathrm{Br} \text {. } \\
\text { XXXII. SOLANACEAE }\end{array}$ & Akanda & Shrub & F1.\& Fr.: Apr.-May & MR 27 \\
\hline 73. Cestrum nocturnum L. & Hasnahena & Shrub & F1.\& Fr.: Jan.-Dec. & MR 76 \\
\hline 74. Datura metel L. & Dhutra & Shrub & F1.\& Fr.: Jul.-Dec. & MR 54 \\
\hline 75. Nicotiana plumbaginifolia Viv. & Bantamak & Herb & F1.\& Fr.: Mar.-Jun. & MR 101 \\
\hline 76. Physalis minima $\mathrm{L}$. & Kopalphutki & Herb & F1.\& Fr.: Sep.-Dec. & MR 55 \\
\hline 77. Solanum nigrum L. & Titbegun & Herb & F1.\& Fr.: Jan.-Dec. & MR 56 \\
\hline $\begin{array}{l}\text { 78. Solanum filicifolium } \mathrm{L} \text {. } \\
\text { XXXIII. CONVOLVULACEAE }\end{array}$ & Katabegun & Shrub & F1.\& Fr.: Jan.-Dec. & MR 30 \\
\hline 79. Ipomoea aquatica Frosk. & Kolmi & Climber & F1.\& Fr.: Nov.-Apr. & MR 67 \\
\hline $\begin{array}{l}\text { 80. Ipomoea fistulosa Martins ex Choisy. } \\
\text { XXXIV. BORAGINACEAE }\end{array}$ & Dholkolmi & Shrub & F1.\& Fr.: Jan.-Dec. & MR 58 \\
\hline $\begin{array}{l}\text { 81. Heliotropium indicum } \mathrm{L} . \\
\text { XXXV. VERBENACEAE }\end{array}$ & Hatisur & Herb & F1.\& Fr.: Jan.-Dec. & MR 63 \\
\hline 82. Clerodendrum viscosum Vent. & Vhat & Shrub & F1.\& Fr.: Feb.-Mar. & MR 60 \\
\hline 83. Lantana camara L. & Lantana & Shrub & F1.\& Fr.: Sep.-Dec. & MR 82 \\
\hline $\begin{array}{l}\text { 84. Vitex negundo L. } \\
\text { XXXVI. LAMIACEAE }\end{array}$ & Nishinda & Shrub & F1.\& Fr.: May-Apr. & MR 96 \\
\hline 85. Leucas lavendulaefolia $\mathrm{Sm}$. & Setodron & Herb & F1.\& Fr.: Aug.-Apr. & MR 62 \\
\hline $\begin{array}{l}\text { 86. Ocimum sanctum L. } \\
\text { XXXVII. OLEACEAE }\end{array}$ & Tulshi & Shrub & F1.\& Fr.: Apr.-Jun. & MR 99 \\
\hline $\begin{array}{l}\text { 87. Nyctanthes arbor-tristis } \mathrm{L} \text {. } \\
\text { XXXVIII. ACANTHACEAE }\end{array}$ & Sheuli & Shrub & F1.\& Fr.: Apr.-Feb. & MR 02 \\
\hline 88. Andrographis paniculata (Burm.f.)Wall exNees. & Kalomegh & Herb & F1.\& Fr.: Jan.-Mar. & MR 54 \\
\hline $\begin{array}{l}\text { 89. Adhatoda vasica Nees in Wall. } \\
\text { XXXIX. RUBIACEAE }\end{array}$ & Basak & Shrub & F1.\& Fr.: May-Jun. & MR 03 \\
\hline 90. Anthocephalus chinensis (Lamk.) Rich. ex Walp. & Kadam & Tree & F1.\& Fr.: Dec.-Jul. & MR 63 \\
\hline 91. Gardenia jasminoides Ellis. & Gandharaj & Shrub & F1.\& Fr.: & MR 53 \\
\hline $\begin{array}{l}\text { 92. Ixora coccinea } \mathrm{L} \text {. } \\
\text { XL. ASTERACEAE }\end{array}$ & Rangan & Shrub & F1.\& Fr.: Jan.-Dec. & MR 48 \\
\hline 93. Eclipta alba $\mathrm{L}$. & Kalokeshi & Herb & F1.\& Fr.: Jul.-Sep. & MR 65 \\
\hline 94. Mikania cordata (Burm.f.) Robinson & Asamlata & Climber & F1.\& Fr.: Oct.-Feb. & MR 89 \\
\hline 95. Tridax procumbens $\mathrm{L}$. & Tridhara & Herb & F1.\& Fr.: Jan.-Dec. & MR 37 \\
\hline 96. Tagetes patula $\mathrm{L}$. & Ghada & Herb & F1.\& Fr.: Dec.-Mar. & MR 01 \\
\hline $\begin{array}{l}\text { 97. Vernonia patula (Dryand.) Merril. } \\
\text { LILIOPSIDA }\end{array}$ & Kuksim & Herb & F1.\& Fr.: Jan.-Dec. & MR 68 \\
\hline XLI. POACEAE & & & & \\
\hline $\begin{array}{l}\text { 98. Bambusa balcooa } \text { Schard. } \\
\text { XLII. ARECACEAE }\end{array}$ & Bansh & Tree & F1.\& Fr.: & MR 88 \\
\hline 99. Areca catechu L. & Khair & Tree & F1.\& Fr.: Mar.-May & MR 102 \\
\hline 100. Borassus flabellifer $\mathrm{L}$. & Tal & Tree & F1.\& Fr.: Mar.-Apr. & MR 71 \\
\hline 101. Cocos nucifera L. & Narikel & Tree & F1.\& Fr.: Mar.-Jul. & MR 103 \\
\hline $\begin{array}{l}\text { 102. Phoenix sylvistris L. } \\
\text { XLIII. ARACEAE }\end{array}$ & Khejur & Tree & F1.\& Fr.: Feb.-Apr. & MR 104 \\
\hline 103. Alocasia indica (Roxb.) Schott. & Mankachu & Herb & F1.\& Fr.: Aug.-Oct. & MR 74 \\
\hline $\begin{array}{l}\text { 104. Colocasia esculenta (L.) Schott. } \\
\text { XLIV. LILIACEAE }\end{array}$ & Kochu & Herb & F1.\& Fr.: Dec.-Mar. & MR 84 \\
\hline 105. Aloe barbadensis Mill. & Ghritakumari & Herb & F1.\& Fr.: Jul.-Dec. & MR 91 \\
\hline 106. Smilax zeylanica $\mathrm{L}$. & Kumarica & Climber & F1.\& Fr.: Jul.-Oct. & MR 86 \\
\hline
\end{tabular}

Jan.=January, Feb.=February, Mar.=March, Apr.=April, May=May, Jun.=June, Jul.=July, Aug.=August, Sep.=September, Oct.=October, Nov.=November, Dec. $=$ December

Table 2. Analytical data of species diversity of three graveyards

\begin{tabular}{cccc}
\hline Study area & No. of species & Percentage & Total number of species \\
\hline Tikapara & 98 & $92.45 \%$ & 106 \\
\hline
\end{tabular}




\begin{tabular}{|c|c|c|c|c|}
\hline Hatem Khan & 79 & $74.52 \%$ & & \\
\hline Meherchandi & 43 & $40.56 \%$ & & \\
\hline Family & No of genera & No of species & Percentage & Total number of family \\
\hline Moraceae & 3 & 6 & $13.63 \%$ & 44 \\
\hline Caesalpiniaceae & 4 & 5 & $11.36 \%$ & 44 \\
\hline Mimosaceae & 3 & 5 & $11.36 \%$ & 44 \\
\hline Euphorbiaceae & 5 & 6 & $13.63 \%$ & 44 \\
\hline Rutaceae & 5 & 5 & $11.36 \%$ & 44 \\
\hline Solanaceae & 5 & 6 & $13.63 \%$ & 44 \\
\hline Asteraceae & 5 & 5 & $11.36 \%$ & 44 \\
\hline Arecaceae & 4 & 4 & $9.09 \%$ & 44 \\
\hline
\end{tabular}

Table 4. Medicinal plants used by local people of Rajshahi city

\begin{tabular}{|c|c|c|c|c|}
\hline $\begin{array}{l}\text { Sl. } \\
\text { no. }\end{array}$ & Plant species & Family name & Part(s) used & Diseases to be treated \\
\hline 1 & Achyranthes aspera $\mathrm{L}$. & Amaranthaceae & $\mathrm{L}, \mathrm{B}, \mathrm{F}$ & Sciatica, abortion, eczema and wound. \\
\hline 2 & Adhatoda vasica Wall in Nees. & Acanthaceae & $\mathrm{L}, \mathrm{B}$ & Cough, asthma, vomiting and worm \\
\hline 3 & Alstonia scholaris $\mathrm{Br}$. & Apocynaceae & $\mathrm{B}, \mathrm{LA}$ & Fever, rheumatism, dysentery and earache. \\
\hline 4 & Amaranthus spinosus $\mathrm{L}$. & Amaranthaceae & WP & Asthma and cold fever. \\
\hline 5 & Aloe vera $\mathrm{L}$. & Liliaceae & $\mathrm{L}, \mathrm{M}$ & Beautification, tonic, anthelmintic, wound and itches. \\
\hline 6 & Aegle marmelos (L.) Correa. & Rutaceae & $\mathrm{F}, \mathrm{L}$ & $\begin{array}{l}\text { Babies dysentery, digestive, tonic, stomachic, abscess } \\
\text { and fever. }\end{array}$ \\
\hline 7 & Annona squamosa $\mathrm{L}$. & Annonaceae & $\mathrm{L}, \mathrm{B}, \mathrm{R}, \mathrm{F}$ & Dysentery, tonic, diarrhea, abscess. \\
\hline 8 & $\begin{array}{l}\text { Andrographis paniculata (Burm.f.) } \\
\text { Wall. ex Nees }\end{array}$ & Acanthaceae & $\mathrm{L}, \mathrm{WP}$ & $\begin{array}{l}\text { Wound, ringworm, itches, fever, dysentery, diarrhea and } \\
\text { tonic. }\end{array}$ \\
\hline 9 & $\begin{array}{l}\text { Anthocephalus chinensis (Lamk.) } \\
\text { Rich. ex Walp. }\end{array}$ & Rubiaceae & $\mathrm{L}, \mathrm{B}$ & Tonic, wound and mouth ulcer. \\
\hline 10 & Artocarpus heterophyllus Lamk. & Moraceae & $\mathrm{L}, \mathrm{B}$ & Wound, asthma, itches and menstrual problems. \\
\hline 11 & Argemone mexicana $\mathrm{L}$. & Papaveraceae & $\mathrm{S}, \mathrm{R}, \mathrm{LA}$ & $\begin{array}{l}\text { Fever, cold, jaundice, diabetes, tonic, diuretic, pain } \\
\text { killer, wound, skin disease and itches. }\end{array}$ \\
\hline 12 & Asparagus racemosus $\mathrm{L}$. & Liliaceae & $\mathrm{B}, \mathrm{R}$ & $\begin{array}{l}\text { Tonic, blood dysentery, diabetes, jaundice, diarrhea, } \\
\text { promotes lactation in mother, wound and itches. }\end{array}$ \\
\hline 13 & Azadirachta indica A. Juss. & Meliaceae & $\mathrm{L}, \mathrm{F}$ & Worm, eczema, ringworm, itches and lice killer. \\
\hline 14 & Bombax ceiba $\mathrm{L}$. & Bombacaceae & $\mathrm{B}, \mathrm{R}$ & $\begin{array}{l}\text { Wound, itches, dysentery, diarrhea, excessive menstrual } \\
\text { discharge and diabetes. }\end{array}$ \\
\hline 15 & Cassia alata $\mathrm{L}$. & Caesalpiniaceae & $\mathrm{L}$ & Ringworm, eczema and dad. \\
\hline 16 & Cassia sophera $\mathrm{L}$. & Caesalpiniaceae & $\mathrm{L}, \mathrm{R}$ & Fever, diuretic, ringworm and sore. \\
\hline 17 & Cajanus cajan (L.) Huth. & Fabaceae & $\mathrm{L}, \mathrm{R}$ & Jaundice and diabetes. \\
\hline 18 & Centella asiatica (L.) Urban & Apiaceae & $\mathrm{L}, \mathrm{WP}$ & Dysentery, headache, itches and eczema. \\
\hline 19 & Citrus grandis (L.) Osbeck. & Rutaceae & $\mathrm{B}, \mathrm{F}$ & Cough and influenza. \\
\hline 20 & Coccinia grandis $\mathrm{L}$. & Cucurbitaceae & $\mathrm{L}, \mathrm{R}$ & Fever, diabetes, cough, asthma and dysentery. \\
\hline 21 & Cocos nucifera $\mathrm{L}$. & Arecaceae & $\mathrm{R}, \mathrm{F}$ & $\begin{array}{l}\text { Cooling, cholera, diarrhea, dysentery, diuretic and } \\
\text { menstrual disease. }\end{array}$ \\
\hline 22 & Calotropis procera $\mathrm{Br}$. & Asclepiadaceae & $\mathrm{L}, \mathrm{R}, \mathrm{LA}$ & Pain, dysentery and injury, \\
\hline 23 & Datura metel L. & Solanaceae & $\mathrm{L}$ & Wound and earache. \\
\hline 24 & Dalbergia sissoo Roxb. & Fabaceae & $\mathrm{L}, \mathrm{W}$ & Gonorrhoea, wound, itches, abscess and vomiting. \\
\hline 25 & Eclipta alba (L.) Hassk. & Asteraceae & $\mathrm{L}, \mathrm{WP}$ & $\begin{array}{l}\text { Wound, itches, skin disease, colour of hairs, jaundice, } \\
\text { asthma and gall bladder stone. }\end{array}$ \\
\hline 26 & Erythrina variegata $\mathrm{L}$. & Fabaceae & $\mathrm{L}, \mathrm{B}, \mathrm{R}$ & $\begin{array}{l}\text { Blood dysentery, earache, toothache, fever and } \\
\text { menstrual discharges. }\end{array}$ \\
\hline 27 & Feronia limonia (L.) Sw. & Rutaceae & $\mathrm{L}, \mathrm{F}$ & $\begin{array}{l}\text { Dysentery, vomiting, blood dysentery, tonic, diuretic and } \\
\text { diarrhea. }\end{array}$ \\
\hline 28 & Ficus benghalensis $\mathrm{L}$. & Moraceae & BU, LA & Diarrhea, dysentery, rheumatism and toothache. \\
\hline
\end{tabular}




\begin{tabular}{|c|c|c|c|c|}
\hline 29 & Glycosmis pentaphylla (Retz.) A. DC. & Rutaceae & $\mathrm{L}, \mathrm{F}$ & $\begin{array}{l}\text { Dysentery, cough, fever, jaundice, rheumatism, eczema } \\
\text { and skin disease. }\end{array}$ \\
\hline 30 & Justicia gendarussa L. & Acanthaceae & $\mathrm{L}$ & Asthma, rheumatism, wound and itches. \\
\hline 31 & Kalanchoe pinnata (Lam.) Pers. & Crassulaceae & $\mathrm{L}$ & $\begin{array}{l}\text { Cold, cough, diabetes, diuretic, blood dysentery and } \\
\text { wound. }\end{array}$ \\
\hline 32 & Lawsonia inermis $\mathrm{L}$. & Lythraceae & $\mathrm{L}$ & Wound, burning sensation, dandruff and foot sore. \\
\hline 33 & Mangifera indica $\mathrm{L}$. & Anacardiaceae & $\mathrm{L}, \mathrm{B}, \mathrm{SD}$ & $\begin{array}{l}\text { Burning sensation, fever, toothache, dysentery and } \\
\text { diabetes. }\end{array}$ \\
\hline 34 & Mimosa pudica $\mathrm{L}$. & Mimosaceae & $\mathrm{L}, \mathrm{SH}, \mathrm{R}$ & Fever and dysentery. \\
\hline 35 & Ocimum sanctum $\mathrm{L}$. & Lamiaceae & $\mathrm{L}, \mathrm{R}$ & $\begin{array}{l}\text { Cold, cough, itches, ringworm, earache, wound and } \\
\text { fever. }\end{array}$ \\
\hline 36 & Oxalis corniculata L. & Oxalidaceae & $\mathrm{L}$ & $\begin{array}{l}\text { Cough, scabies, itches, dysentery, anemia, piles, } \\
\text { dyspepsia and fever. }\end{array}$ \\
\hline 37 & Punica granatum $\mathrm{L}$. & Punicaceae & $\mathrm{F}, \mathrm{R}$ & Dysentery, diarrhea and worm. \\
\hline 38 & Phyllanthus emblica L. & Euphorbiaceae & $\mathrm{F}$ & $\begin{array}{l}\text { Tonic, diuretic, vomiting, cold, cough, burning } \\
\text { sensation, dysentery, anemia, jaundice, dyspepsia and } \\
\text { hair tonic. }\end{array}$ \\
\hline 39 & Psidium guajava (L.) Bat. & Myrtaceae & $\mathrm{L}, \mathrm{S}, \mathrm{F}$ & Dysentery, toothache, vomiting and diarrhea. \\
\hline 40 & Rauvolfia serpentina Benth. & Apocynaceae & $\mathrm{R}$ & $\begin{array}{l}\text { Blood pressure, tonic, diarrhea, dysentery, colic and } \\
\text { fever. }\end{array}$ \\
\hline 41 & Ricinus communis $\mathrm{L}$. & Euphorbiaceae & $\mathrm{L}, \mathrm{SD}$ & Headache and rheumatism. \\
\hline 42 & Syzygium cumini (L.) Skeel. & Myrtaceae & $\mathrm{B}, \mathrm{SD}$ & Dysentery, wound and diabetes. \\
\hline 43 & Smilax zeylanica $\mathrm{L}$. & Liliaceae & $\mathrm{R}$ & Blood dysentery and abscess. \\
\hline 44 & Tamarindus indica $\mathrm{L}$. & Caesalpiniaceae & $\mathrm{L}, \mathrm{F}$ & $\begin{array}{l}\text { Burning sensation, dyspepsia, appetite, digestive, cold, } \\
\text { tonic and dysentery. }\end{array}$ \\
\hline 45 & Terminalia chebula (Gaertn.) Retz. & Combretaceae & $\mathrm{F}$ & $\begin{array}{l}\text { Wound, worm, rheumatism, vomiting, urinary disease } \\
\text { and dysentery. }\end{array}$ \\
\hline 46 & Terminalia arjuna (Roxb.)Wt. \& Arn & Combretaceae & $\mathrm{L}, \mathrm{SH}$ & Heart disease, burning sensation and dyspepsia. \\
\hline 47 & Vitis quadrangularis Wall. & Vitaceae & $\mathrm{S}$ & Bone fracture and gout. \\
\hline 48 & Vitex negundo L. & Verbenaceae & $\mathrm{L}, \mathrm{R}$ & Cold, cough, asthma, tonic, fever and diuretic. \\
\hline 49 & Zizyphus mauritiana Lamk. & Rhamnaceae & $\mathrm{L}, \mathrm{S}$ & Blood dysentery, wound and headache. \\
\hline
\end{tabular}

$\mathrm{L}=$ Leaf, $\mathrm{S}=$ Stem, $\mathrm{R}=$ Root, $\mathrm{SD}=$ Seed, $\mathrm{LA}=$ Latex, WP=Whole plant, $\mathrm{SH}=$ Shoot, $\mathrm{F}=$ Fruit, $\mathrm{B}=$ Bark, BU=Bud, W=Wood

Table 5. Analysis of the data based on habit showed that leading medicinal plants species

\begin{tabular}{cccc}
\hline Habit & No. of species & Percentage & Total number of species \\
\hline Herbs & 11 & $22.44 \%$ & 49 \\
Shrubs & 9 & $18.36 \%$ & 49 \\
Climbers & 7 & $14.28 \%$ & 49 \\
Trees & 23 & $46.93 \%$ & 49 \\
\hline
\end{tabular}

Medicinally important plants: The important medicinal plants in the graveyards of Rajshahi city corporation area were carried out. A total of 49 plant species belonging to 47 genera and 33 families were collected and identified. Most of the local people in the study area are poor are illiterate. In one hand, these people are out of the reach of modern medicines and on other hand, the market price of most available medicines are very expensive. As a result, these medicinal plants are used by them to cure following the diseases, especially for abscess, asthma, abortion, cough, cold, chicken pox, constipation, dysentery, diarrhea, diabetes, eczema, fever, fracture of bone, headache, heart disease, itches, jaundice, menstrual disease, paralysis, piles, skin diseases, snake-bite, sex problems, toothache, vomiting, worm, wound and others. Different plant parts of different spp. are used as medicine for treating various diseases; bark of 15 , leaf of 39 , fruit of 9 , root of 11 , seed of 1 , wood of 1 , latex of 4 , stem of 5 , mucilage of 1 , and whole plant of 3 species were used as medicine (Table 4).

Analysis of the data based on habits showed that leading medicinal plant species $22.44 \%$ belonged to herbs, $18.36 \%$ shrubs, $14.28 \%$ climbers and $46.93 \%$ trees (Table 5 ).

\section{Conclusion}

Angiosperm diversity in the graveyards of Rajshahi city corporation area has been studied. A total of 106 angiosperm species under 91 genera and 44 families were recorded. The graveyards of Tikapara and Hatem Khan were two protected areas. The plants, which grow naturally or planted in these graveyards, were not destroyed by 
someone. That is why huge quantity of plant species was observed here. Its biodiversity was developed. On the other hand Meherchandi graveyard was an open place. It was comparatively a new graveyard. Plants were observed in a lesser extent due to grazing in this graveyard. As a result, biodiversity of Meherchandi graveyard was comparatively less than Tikapara and Hatem Khan Graveyards. A total of 49 medicinal plant species belonging to 47 genera and 33 families were collected and recorded for their use in various ailments.

\section{Acknowledgements}

The author is grateful to the Ministry of Science, Information and Communication Technology, Government People's Republic of Bangladesh for financial support to complete this research work. Thanks are also due to the local people of Rajshahi city for their co-operation and help during the research work.

\section{References}

[1] Ahmed Z U, Begum Z N T, Hassan M A, Khondker M, Kabir S M H, Ahmad M, Ahmed A T A, Rahman A K A and Haque E U(Eds). Encyclopedia of Flora and Fauna of Bangladesh.. Angiosperms; Dicotyledons. Asiat. Soc. Bangladesh, Dhaka. Vol 6-12, 2007-2009.

[2] BBS (Bangladesh Bureau of Statistics). Statistical Year Book of Bangladesh, 23rd edition, Bangladesh Bureau of Statistics, Planning Division, Ministry of Planning Government of Peoples Republic of Bangladesh, Dhaka, 2009.

[3] Cronquist A. An Integrated System of Classification of Flowering Plants. Columbia University Press. New York, 1981.

[4] Hooker JD. Flora of British India. L. Reeve and Co. Ltd. London, Vol 1-7, 1961.

[5] Khan MS, Rahman MM, Huq AM, Mia MMK and Hassan MA. Assessment of Biodiversity of Teknaf Game Reserve in Bangladesh focusing on economically and ecologically important plant species. Bangladesh J. Plant Taxon.1994; $1(1): 21-23$.
[6] Khan MS and Huq AM. The vascular flora of Chunati wildlife Sanctuary in South Chittagong. Bangladesh J. Plant Taxon. 2001; 8(1): 47-64.

[7] Kirtikar KR and Basu BD. Indian Medicinal Plants. Lalit Mohan Basu, Alhabad, India.,Vol 1-4, 1987.

[8] [8] Pasha MK and Uddin SB. Dictionary of Plant Names of Bangladesh (Vascular Plants). Janokalyan Prokashani. Chittagong, Dhaka, Bangladesh. 2013; pp. 1-434.

[9] Prain D. Bengal Plants. Botanical Survey of India, Calcutta. India. Vol 1-2, 1963.

[10] Rahman AHMM, Islam AKMR and Zaman ATMN. Studies on herbaceous plant species in the graveyard areas of Rajshahi City. Plant Environ. Dev. 2007; 1(1): 57-60.

[11] Rahman MO and Hassan MA. Angiospermic flora of Bhawal National Park, Gazipur (Bangladesh). Bangladesh J. Plant Taxon. 1995; 2(1\&2): 47-79.

[12] Rahman MO, Uddin MZ, Tutul E, Begum M and Hassan MA. Additions to the angiospermic flora of Runctia Sal forest, Bangladesh. Bangladesh J. Plant Taxon. 2010; 17(2): 171-185.

[13] Rahman MO, Antara MT, Begum M and Hassan MA. Floristic diversity of Dhamrai Upazilla of Dhaka with emphasis on medicinal plants. Bangladesh J. Bot. 2012; 41(1): 71-85.

[14] Tutul E, Uddin MZ, Rahman MO and, Hassan MA. Angiospermic flora of Runctia Sal forest, Bangladesh. I. Liliopsida (Monocots) Bangladesh J. Plant Taxon. 2009; 6(1): 83-90.

[15] Tutul E, Uddin MZ, Rahman MO and Hassan MA. Angiospermic flora of Runctia Sal forest, Bangladesh. II. Magnoliopsida (Dicots) Bangladesh J. Plant Taxon. 2010; 17(1): 33-53.

[16] Uddin SB and Rahman MA. Angiospermic flora of Himchari National Park, Cox's Bazar, Bangladesh. Bangladesh J. Plant Taxon. 1999; 6(1): 31-68.

[17] Uddin MZ and Hassan MA. Angiosperm diversity of Lawachara National Park (Bangladesh). Bangladesh J. Plant Taxon. 2010; 17(1): 9-22. 